\title{
Design and construction of fascia lata aortic valve prostheses
}

\author{
KENNETH G. REID \\ Nuffield Department of Surgery, Oxford
}

\begin{abstract}
Current techniques for constructing fascia lata aortic valves result in a valve in which redundant cusp tissue is inadvertently incorporated in the proximal part of the cusp. This alters the flow character of the valve and produces a significant stenosis. An alternative technique, based on the normal anatomy of the aortic valve, is suggested which eliminates this fault. The technique is simple and can be used at the time of operation.
\end{abstract}

The recent literature indicates that the use of autologous fascia lata as a heart valve cusp substitute material is rapidly becoming popular surgical practice and there are now well-recognized methods for readily fashioning heart valves of this type at the time of operation (Senning, 1967 ; Ionescu and Ross, 1969 ; Ionescu, Ross, Deac, Grimshaw, Taylor, Whitaker, and Wooler, 1970). The majority of surgeons at present favour a technique in which fascia is sewn onto a Dacroncovered titanium stent for rapid subsequent insertion and ease of manufacture. The advantages of using fascia lata in a prosthetic valve have been detailed (Ionescu and Ross, 1969; Ionescu et al., 1970), but especially important is that, unlike alternatives, fascia does not of itself impose design limitations on the surgeon constructing a valve. It is particularly for this reason that fascia lata is an attractive material since it may be preformed to the most ideal shape. It is important, therefore, that current valve design faults are identified and, where possible, avoided. There are important theoretical and practical objections to the fascial valves produced by the methods recently outlined, since they result in a valve whose 'anatomy' and flow characteristics are some way short of the ideal. An alternative technique is outlined which eliminates these inherent faults and which is based on the study of the anatomy of fresh aortic valves and their simulation in a pulse duplicator.

It has been shown that normal aortic valve cusps have no recoil tendency in the fresh state but lie collapsed in an open position (Reid, 1970), that at peak systole the valve cusps are widely open and in important relation to a sinus ridge, and that the velocity profile across the aorta just distal to the ridge is flat (Bellhouse and Reid, 1969 ; Bellhouse, Bellhouse, and Reid, 1968). The reproduction of this aortic root geometry, in particular of cusps that conform to a cylinder when fully open, in a pulsed analogue has shown that the valve closure mechanism depends on the formation of trapped vortices in the sinus of Valsalva. The sinuses act as pressure recovery chambers regulating systolic coronary blood flow and initiating valve closure in late systole (Reid and
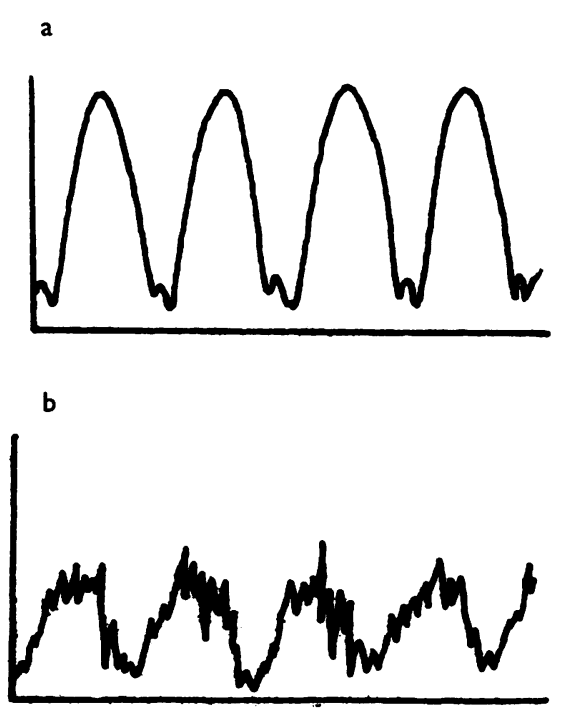

FIG. 1. Instantaneous velocity traces of (a) model aortic valve constructed so that the cusps when fully open conform to a cylinder. Note absence of small fluctuations (b) aortic valve showing effect of significant stenosis. Note rapid random fluctuations indicating turbulence. 
Bellhouse, 1968). Any element of valvular stenosis produces an abnormal flow pattern in the aortic root, compromising smooth efficient closure and reducing coronary blood flow (Reid and Bellhouse, 1968) (Fig. 1).

The present technique of fashioning valves in the closed position, using either the 'three-finger' principle (Ionescu) or a tripedal sprung clamp (Ross), produces a valve whose internal diameter varies along its length by up to $15 \%$, which is significantly stenosed, and whose haemodynamic function is imperfect. By allowing the closed cusp to conform to the shape of the finger or a preformed mould of similar geometry, redundant cusp tissue is inadvertently incorporated within the proximal portion of the cusp. The result is to create ballooning when the cusp is fully open. This effect may also be readily appreciated if a moistened finger is allowed to run up the inner aspect of the cusp using slight pressure. Further evidence of this is provided if an excised fascial cusp, fixed in formalin, is laid onto a flat surface, when, unlike a normal cusp treated in the same way, it always buckles in its proximal portion. The outline of such a cusp is flask- rather than cup-shaped (Fig. 2).

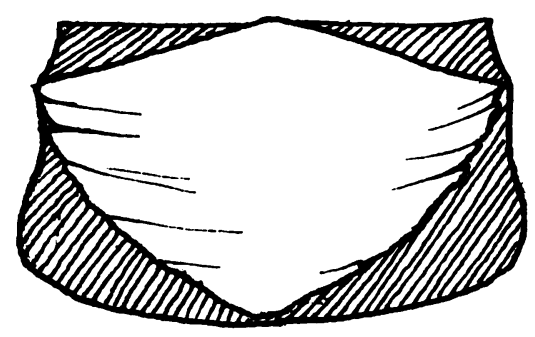

FIG. 2. Outline of normal aortic cusp when excised and, superimposed, the outline of a fascial cusp (shaded) when constructed by the three-finger method.

An additional fault centres on the current method of handling the commissures. The present technique of sewing the commissure within the diameter of the valve further accentuates the stenosis produced by indenting the closed cusps. It is evident that moving the commissures to a point outside the internal diameter will leave the primary cusp fault unresolved.

These objections can be met by constructing a valve like the natural valve in the open position using a central cylindrical mould and separate portions of fascia. Only in this way can the internal

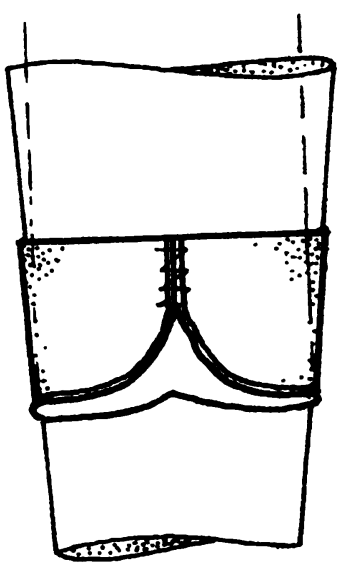

FIG. 3. Method for constructing fascial valves using a stent and a cylindrical divergent mould. Note reduction in height of commissure.

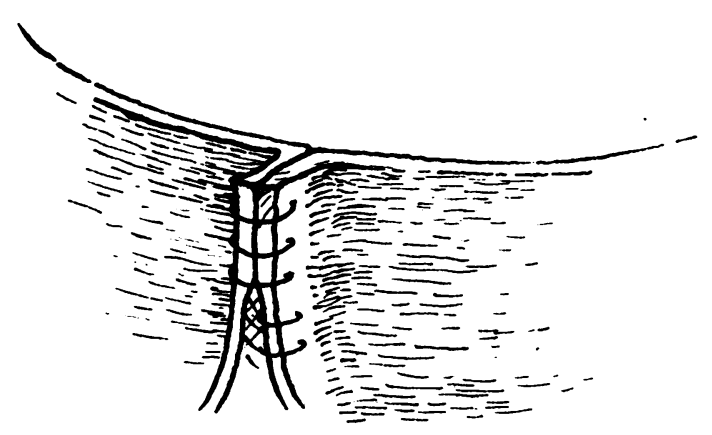

FIG. 4. Detail drawing of method of arranging commissure so as to ensure accurate apposition of cusps.

diameter be controlled along the length of the valve. It is important to stress that the shape assumed by the valve cusps in the closed position is of little importance provided that there is adequate coaption in diastole. Experimental studies underline that it is the cusp configuration when fully open that is all important to the closure mechanism and for ensuring systolic coronary blood flow (Reid, 1969). If anxiety exists about adequate apposition of the cusps in diastole, they are constructed around a cylinder that is slightly diverging, so that the cusp tip outlet radius exceeds the cusp hinge inlet radius by a matter of millimetres only (Fig. 3). At the same time the height of the commissural stent is reduced so that the cusps 
extend for a short distance (about $4 \mathrm{~mm}$.) above it where they are sewn together. In this way the internal diameter is controlled to avoid stenosis, and accurate cusp apposition is ensured (Fig. 4).

\section{REFERENCES}

Bellhouse, B. J., and Reid, K. G. (1969). Fluid mechanics of the aortic valve. Brit. Heart J., 31, 391.

- Bellhouse, F. H., and Reid, K. G. (1968). Fluid mechanics of the aortic root with application to coronary flow. Nature (Lond.). 219, 1059.
Ionescu, M. I., and Ross, D. N. (1969). Heart valve replacemer with autologous fascia lata. Lancet, $2,335$.

Deac, R., Grimshaw, V. A., Taylor, S. H., Whitaker, W? and Wooler, G. H. (1970). Aut Reid, K. G. (1969). Functional anatomy of the aortic root. Circula
lation, 40, Suppl. 3, p. 168 .

- (1970). The anatomy of the sinus of Valsalva. Thorax, 25, 79

- and Bellhouse, B. J. (1968). The mechanism of aortic valve closure and the function of the sinus of Valsalva. In ProcEuropean Congress of Cardiovascular Surgeons, Royal College of Surgeons, London. Minerva Medica, Milan.

Senning, A. (1967). Fascia lata replacement of aortic valves. thorac. cardiovasc. Surg., 54, 465. 